\title{
Direct and indirect perception of size
}

\author{
JOEL NORMAN \\ University of Haifa, Haifa 31999, Israel
}

\begin{abstract}
Three experiments, using a reaction time paradigm, examine the direct (stimulus bound) and indirect (mediational inference) approaches to size perception. Subjects determine which of two stimuli is the larger when the two can be at different egocentric distances. The effects of two variables on reaction times are examined-distal ratio, the ratio of physical sizes of the stimuli, and proximal ratio, the ratio of the angular projections of the stimuli on the retina. In Experiment 1, both ratios are found to affect reaction times, with the proximal ratios yielding the larger effect, more in line with the predictions of the indirect approach. But the results of Experiments 2 and 3 indicate that distance is taken into greater account, the more similar the distal sizes of the stimuli. In one stimulus condition, distance appears not to affect reaction times. It is suggested that direct size perception occurs for large stimulus differences, indirect size perception for smaller differences. The identical results of the two experiments, one with and one without texture, point to some variable other than texture occlusion or interception as the stimulus for direct size perception. Some aspect of distance from the eye-level plane is suggested as an alternative.
\end{abstract}

Research on size perception has expended much effort on explaining the invariance of perceived size with variable object distance, a perceptual capacity that is usually called "size constancy." The visual system is quite good at determining the veridical or distal size of an object in spite of the fact that its retinal counterpart, the proximal stimulus, varies in size with changes in distance. Several theories, or models, have been proposed to explain size constancy, as well as the other constancies. These can be dichotomized into what have been called sensory vs. cognitive theories (e.g., Beck, 1972; Hochberg, 1971), proximal vs. algorithmic (Epstein, 1977), or direct vs. indirect (e.g., Turvey, 1977). The directindirect labels will be used here, but no attempt will be made to explore their deeper, philosophical connotations (see, e.g., Shaw \& Bransford, 1977). Direct theories, as used here, refer to those theories which emphasize the content of the visual stimulus as the main, if not sole, determiner of what is perceived. The indirect theories, on the other hand, assume the operation of mediational and interpretative mechanisms in the perceiver, emphasizing the equivocality of the visual stimulus. Within the domain of space perception, the indirect theories are currently the more fashionable, not only because of their aristocratic ancestry (Helmholtz, 1925), but also because of a great deal of empirical evidence that

This research was supported in part by a grant from the Faculty of Social Sciences, University of Haifa. The author would like to thank David Navon for his insightful comments, Jacob Shtulbach and Amy Shamir for their assistance in various phases of the research, and Abraham Papo for his able technical assistance. Requests for reprints should be addressed to Joel Norman, Department of Fsychology, University of Haifa, Haifa 31999, Israel. corroborates them (see, e.g., Epstein, 1973; Rock, 1977). On the other hand, the direct approach continues to be championed by Gibson (1959, 1966, 1979), with support from a few other sources (e.g., Mace, 1977; Turvey, 1977).

The invariance of perceived size with distance variant is ascribed by indirect theories to a hypothetical, unconscious, and very rapid process that takes distance into account (e.g., Epstein, 1973). This process utilizes proximal size and distance cues to calculate or infer the resultant veridical distal size. The interaction between proximal size information and perceived or registered distance in yielding distal size can take on several forms (see Oyama, 1977; Sternberg, Note 1), but all variants involve perceived or registered distance in some way. In contrast, Gibson's direct approach ascribes a much richer and unequivocal role to the visual stimulus, leaving the perceiver with the task of extracting the relevant information from the optic array. How can such an approach account for size constancy without positing some ancillary mechanisms that take distance into account? Gibson $(1959,1979)$ suggests that the size of an object could be given by the number of texture elements on the ground that it intercepts or occludes. Hochberg $(1971,1974)$ calls this the texture-scale size cue, an example of higher order stimuli, and shows how perceived size and perceived distance need not be related in this theory. In his recent book, Gibson (1979) argues against a cue theory, preferring to view texture occlusion as but one example of the invariant ratios and relations that serve as information for direct perception.

The main aim of the present set of experiments was to examine the effects of stimulus distance 
on size perception by a reaction time paradigm, in which the subjects would have to determine which of two stimuli was the larger when the two could be at different egocentric distances. The underlying assumption in the use of reaction time as a dependent variable is that the durations of the reaction times reflect the difficulty and complexity of the processing involved.

Reaction time was used as the dependent variable in two studies of size perception by Broota and Epstein (1973; Epstein \& Broota, 1975). The first study consisted of four experiments, two on size and two on distance. In brief, their main finding was that size reaction times increased linearly with increased viewing distance, egocentric distance, but that there was no systematic effect of egocentric distance on distance reaction times. They were hard pressed to explain these findings, but a second study (Epstein \& Broota, 1975) yielded results which, they suggested, threw light on their earlier findings. In that study, size judgment reaction times were obtained under objective and phenomenal size instructions (Epstein, 1963; also see Carlson, 1977). Only the objective instructions yielded the previously found linear increase in reaction time with increasing egocentric distance, while the phenomenal instructions showed no effect of distance. This led Epstein and Broota to suggest that their reaction-time differences occurred at some postperceptual decision stage. But these two experiments raise some procedural problems that render their conclusions tenuous. In the four experiments of the earlier study, the subjects had to make a binary response ("same", vs. "different," or "yes" vs. "no"), whereas subjects in these two experiments were presented with a single response button and were required to report an estimate of size while simultaneously pressing the response button. With such a procedure, measured reaction times are not necessarily related to the perception of size. Perhaps this is why the overall mean reaction time under the phenomenal instructions was very short $(235 \mathrm{msec})$.

The present study sought to create an experimental situation in which the predictions of the direct and indirect theories would be different. The initial idea was to present subjects with two objects, red squares, at different distances in a well-illuminated and richly textured environment, and require a binary reaction time response indicating which square was larger. The direct and indirect theories yield different predictions as to which stimulus situation will be the more complex. According to the direct theory, the visual system could determine the sizes without taking distance into account, perhaps utilizing the number of texture elements occluded. The main variable determining the complexity of the discrimination according to this view should be the simi- larity of the objective distal sizes of the two squares, independent of their egocentric distance. The ratio of objective sizes, or distal ratio (smaller to larger), should be the primary variable affecting reaction time. The closer this ratio is to 1.0 (both squares the same size), the higher the expected reaction time. The distal ratio is, of course, not affected by the distance of the two squares. Thus, the direct theory predicts that the reaction time should be indifferent to stimulus distances.

The indirect theory, on the other hand, asserts that veridical size perception takes distance into account to resolve equivocal proximal sizes. Thus, the complexity of the size comparison task depends on the specific placement of the two squares on the textured surface. When the larger square is closer than the smaller square, its proximal size is also larger, but when it is farther, its proximal size can be equivalent to, or even smaller than, that of the smaller square. The relative size of the two proximal images will be expressed in terms of the proximal ratio, the ratio of the proximal size of the physically smaller square to that of the physically larger square. When the proximal ratio is equal to, or smaller than, the distal ratio, the relative sizes of the two proximal images are in accord with their true distal sizes. But, as the proximal ratios become larger, they are apt to yield more complex discriminations according to the indirect approach. Thus, for example, in those extreme cases in which the proximal ratio exceeds 1.0 , the proximal image of the larger square is smaller than that of the smaller square. The proximal ratio can be seen to reflect the discrepancy between the relative size of the two proximal images and the relative size of the two distal squares. The greater this discrepancy, the more complex the discrimination required. More complex discriminations should yield longer reaction times. Therefore, it is suggested that the indirect theory predicts increasing reaction times with increasing proximal ratios. In contrast, the direct theory predicts that the reaction times should vary only with the distal ratios and not with the proximal ratios, since the latter result from manipulating the distances of the stimuli involved.

The present study consisted of three experiments. The first experiment explored the effects of the two variables, distal and proximal ratios, on reaction time. Because of its exploratory nature, the design called for many stimulus conditions and relatively few reaction times per condition. In the second experiment, fewer conditions were used, and a simpler paradigm was employed, in which one square, the standard, did not change in size or position throughout the experiment for a given subject. The subject had to respond as rapidly as possible whether the comparison square was larger or smaller than the 
standard. In both Experiments 1 and 2 the two squares were placed on a richly textured surface. The third experiment essentially replicated the second, but with the textured surface replaced by a dullgray, textureless one. Its aim was to see in what way the results of Experiment 2 would differ if a strong distance cue and a possible source of direct perception of size were eliminated.

\section{EXPERIMENT 1}

This experiment aimed at assessing the relative effectiveness of the two variables of interest, distal ratio and proximal ratio, on reaction time. Two squares were placed on a richly textured surface and viewed monocularly. The subjects had to determine which of the two was larger, that on the left or that on the right, and quickly press the appropriate button with the corresponding hand. Four different square sizes were used, and the two members of a displayed pair were always at different egocentric distances.

\section{Method}

Subjects. Eighteen undergraduates at the University of Haifa, with normal or corrected vision, participated in the experiment to fulfill a requirement of an introductory psychology course.

Apparatus. The stimuli were presented on an elongated $(420 \times$ $100 \mathrm{~cm}$ ) and elevated (104 $\mathrm{cm}$ above floor level) table covered with a black-gray-white checkered cloth $(2 \times 2 \mathrm{~cm}$ square size $)$. The subject sat $50 \mathrm{~cm}$ from one end of the table, his head firmly supported by a head- and chinrest. His view of the table was occluded by a wooden screen in which an electronic shutter was mounted. The shutter was placed in line with the midline of the table, with its center $15 \mathrm{~cm}$ higher than table-top level. The subject was positioned so that his right eye was directly behind the shutter; that is, when the shutter was open, the subject had a monocular view of the table top. The backdrop at the far end of the table was of plain lemon-yellow cardboard. Normal fluorescent room lighting was maintained throughout the experiment. The subject responded by pressing one of two buttontype microswitches on which the index fingers of his left and right hands were placed. Reaction times were measured on an electronic counter/timer.

Stimulus conditions. Four stimuli were used throughout the experiment. These were square pieces of red matte Plexiglas which stood erect on the table with the aid of a support hidden from the subject's view. The squares were always presented in pairs, each member of a pair at a different distance from the subject. Four distances were used: $200,250,300$, and $350 \mathrm{~cm}$. The two stimuli were always placed at a constant distance of $5 \mathrm{~cm}$ from the midline of the table, one to the left of the midline and the other to the right. The sizes of the four squares were such that each one would subtend exactly $2 \mathrm{deg}$ of visual angle at one of the four distances employed. The lengths of the edges of the four squares were: $1,69.2 \mathrm{~mm}(2 \mathrm{deg}$ when placed at $200 \mathrm{~cm}$ ); $2,87.3 \mathrm{~mm}(2 \mathrm{deg}$ at $250 \mathrm{~cm}) ; 3,104.7 \mathrm{~mm}(2 \mathrm{deg}$ at $300 \mathrm{~cm})$; and $4,122.2 \mathrm{~mm}(2 \mathrm{deg}$ at $350 \mathrm{~cm})$. The squares were placed not only at the distances at which they subtended $2 \mathrm{deg}$, but also at each of the four distances. Altogether there were 72 possible stimulus conditions. These were the various pairings of two squares of different sizes at different distances $(6$ stimulus pairings $\times 12$ arrangements at the four distances).
Procedure. The subjects were instructed that when the shutter opened they would see two squares of different sizes, one to the left and one to the right. They were required to determine which was larger and respond by pressing the corresponding button. They were urged to respond as quickly as they could without making errors. Following nine practice trials, each subject was presented with two trials on each of the 72 stimulus conditions in a single session, which lasted approximately $1 \mathrm{~h}$. The stimulus conditions were allocated randomly to 16 blocks of 9 each, and sequence effects were counterbalanced over subjects with the aid of a balanced square design. This design called for 16 subjects, but the data of 2 of the original subjects were replaced by those of 2 additional subjects (see below).

A single trial consisted of the following sequence of events. After positioning the two squares on the table, the experimenter gave the subject a "ready" signal and started the automatic sequence. This began with a $200-\mathrm{msec}$ warning tone followed by a 400 -msec interval, at the end of which the shutter opened for $2 \mathrm{sec}$. The electronic clock was activated when the shutter opened and stopped when the subject pressed one of the two microswitch buttons. No feedback about correctness was given to the subjects. The intertrial interval was approximately $10 \mathrm{sec}$; there was a short break at the end of each trial block and a longer break after each group of three blocks.

\section{Results and Discussion}

Two subjects were discarded because they made many errors and showed an error pattern different from that of the other subjects. The same results emerged when these subjects were retested and questioning elicited the information that they had evolved a "theory" of how to respond efficiently, which produced their discrepant data. They were replaced by two additional subjects. The following analyses were carried out on these 16 subjects.

Each of the 16 subjects contributed only two reaction times to each of the 72 conditions, once when the larger stimulus was on the right and once when it was on the left. Fourteen of the 16 subjects were right-handed, but performance with the right hand was not significantly faster than performance with the left hand. The respective means for right and left hands were 591 vs. $600 \mathrm{msec}[\mathrm{t}(71)=1.10$, n.s.]. Thus, it was decided to use the means of these 72 conditions as the basic dependent variable data. Error responses, defined in terms of true distal sizes, were not included in the analysis. The mean error rate was $6.0 \%$, ranging for individual subjects from $.7 \%$ to $13.9 \%$. The mean reaction times on the 72 conditions ranged between 467 and $840 \mathrm{msec}$.

To assess the effects of distal and proximal ratios, product moment correlation coefficients were calculated between them and the 72 mean reaction times. Proximal ratio yielded the highest correlation, .84 , while distal ratio yielded a correlation of .57 . It should be noted, though, that these two variables are not independent; in the present design, the correlation between them is .37. But multiple regression and partial correlation analyses indicate that both variables have an independently significant 

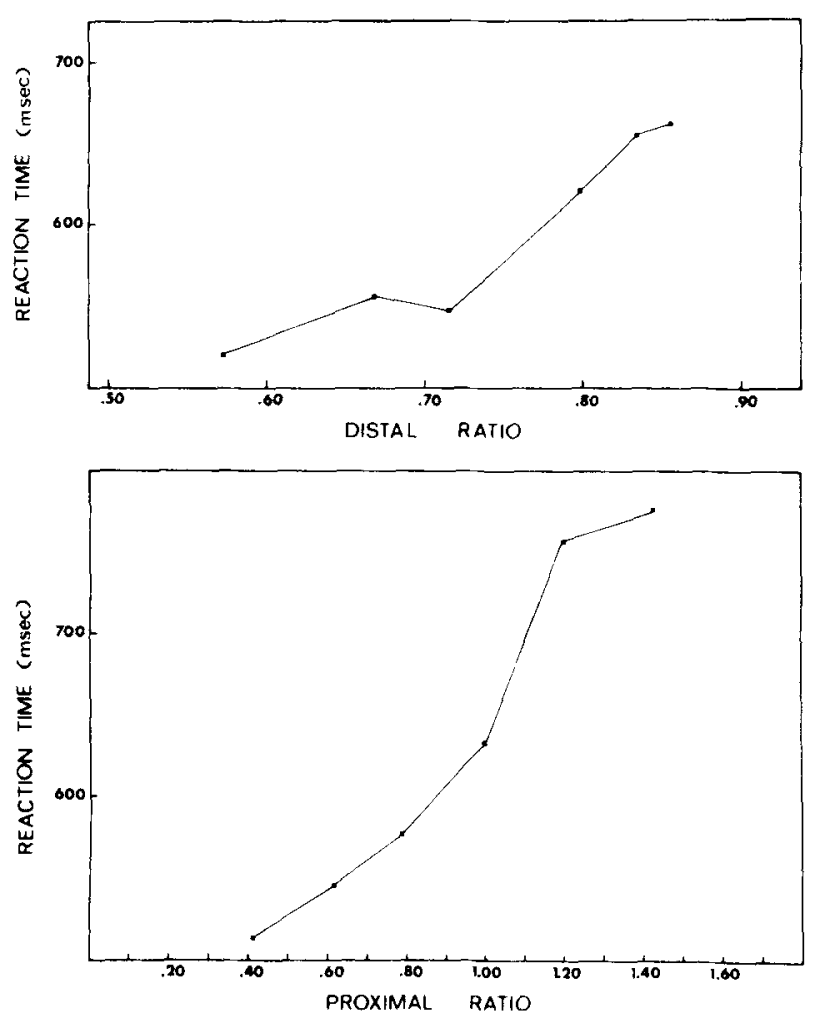

Figure 1. Mean reaction time as a function of distal ratio (upper graph) and proximal ratio (lower graph) in Experiment 1.

effect, with that of the proximal ratio being greater. This can also be seen in Figure 1, in which the effect of both distal and proximal ratio on reaction time are depicted. The values of the proximal ratios have been collapsed onto the means of six ranges (.30-.49, .50-.69, .70-.89, .90-1.09, 1.10-1.29, and 1.30-1.50) in order to present a clear picture of the effect, and also to make it more similar to the distal ratio variable, which takes on only six values. (Under this breakdown, the correlation between proximal ratio and reaction time hardly changes, .83.) Two distance variables yielded near-zero nonsignificant correlation coefficients with reaction time. These were the mean egocentric distance of the two squares and the distance between the two squares.

The finding that the proximal ratio affects the reaction time favors the indirect theory. But the additional finding of an independent effect of the distal ratio points to a more complex situation (see General Discussion).

\section{EXPERIMENT 2}

In this experiment, one square, of intermediate size, remained at a constant position, the "standard," and the subject had to decide if the "comparison," which varied in size and position, was larger or smaller, and respond accordingly. Unlike those in the first experiment, the subjects in the present experiment were motivated to improve their performance and were given immediate feedback after making an error.

\section{Method}

Subjects. Twenty-four undergraduates at the University of Haifa, with normal or corrected vision, participated in the experiment, fulfulling a requirement of an introductory psychology course. None had participated in Experiment 1.

Apparatus. All aspects of the apparatus were identical to those of Experiment 1, with two exceptions. The yellow backdrop at the end of the table was replaced with an artist's accurate rendition of a perspective continuation of the textured surface. The monocular view through the open shutter yielded a convincing representation of the table top's continuing to a (calculated) $35-\mathrm{m}$ distance. The original partition in which the shutter was placed was replaced with a much larger one, which completely blocked the subject's view of the laboratory (ceiling, upper corners). Thus, the subject had no information about the size of the laboratory, except for that gained when the shutter was opened.

Stimulus conditions. The stimulus conditions were based on those of Experiment 1, but with the addition of a fifth distance, $400 \mathrm{~cm}$, and a fifth square, No. $5,139.6 \mathrm{~mm}$ on a side. When square 5 was placed at $400 \mathrm{~cm}$, it subtended $2 \mathrm{deg}$. One further innovation was that the distance of the squares from the midline of the table was not kept constant; instead, the distances were increased with increasing egocentric distance so that their retinal projection was always $1 \mathrm{deg}$. Throughout this experiment, square 3 was the "standard" square, remaining throughout the session at the same position. For half the subjects, the standard was presented at $200 \mathrm{~cm}$, where it subtended $3.0 \mathrm{deg}$ (Group $\mathrm{St} 2$ ); for the other half, it was presented at $400 \mathrm{~cm}$, where it subtended $1.5 \mathrm{deg}$ (Group St4). These two subgroups were further divided so that for half the subjects in each group the standard appeared to the right of the midline and for the other half to the left. Two of the comparison squares, 1 and 2, were smaller than the standard, and two, 4 and 5, were larger. Each of these four squares was presented at each of the five positions, yielding a total of 20 stimulus conditions.

Procedure. The subjects were first shown the standard square and told that it would not change throughout the experiment. They were then shown several examples of the comparison squares and the procedure was explained to them. They had to press a button with one hand if the comparison square was larger than the standard and a second button with the other hand if it was smaller. Half of the subjects pressed "larger" with the right hand, and half with the left hand. They were urged to respond as quickly as they could without making errors. They were then run through a practice block of 20 trials ( a random ordering of the 20 stimulus conditions). After the practice block, the subjects were told that they could accumulate points by improving their reaction times, but would be quite heavily penalized for errors. The experiment proper consisted of 12 blocks of 20 trials each. Each block was a random ordering of the 20 possible stimulus conditions. The order of blocks was counterbalanced over subjects. At the end of each block, the subjects were told how many points they had accumulated or, at times, lost in that block. The subjects were also informed that relatively high monetary prizes would be given to the three subjects who had the highest total scores. The subjects were given feedback after each trial on which they made an error.

\section{Results and Discussion}

The median of 12 reaction times for each subject served as the basic datum. Error responses were not included in the calculation of the medians. The 
mean error rates were $2.8 \%$ for Group St2 and $4.6 \%$ for Group St4. The means of the medians for the two groups are presented in Table 1, together with the respective values of distal ratio and proximal ratio. It can be seen that the same distal ratios yield a fairly wide range of reaction time values, although the means appear to be higher for the higher distal ratios. (See further related analyses in the analyses of variance below.) While proximal ratio yielded a fairly high correlation (.84) with reaction time in Experiment 1, the correlation in the present experiment was only .52. It should be noted that the proximal ratios of the two groups, St2 and $\mathrm{St} 4$, are either doubled or halved and yet the mean reaction times for the same comparison stimuli are quite similar and the correlation between them is quite high, .93 .

It can also be seen in Table 1 that the reaction times for a given comparison stimulus vary systematically with distance. This is also depicted in the two left-hand panels in Figure 2, showing stimulus sizes 1 and 2 separately from stimulus sizes 4 and 5. To further assess these data, an analysis of variance performed with one between-subjects variable, position of standard ( $\mathrm{St} 2$ and $\mathrm{St} 4)$, and two within-subjects variables, distance of comparison square and size

Table 1

Mean Reaction Times (RT) in Milliseconds in Experiment $\mathbf{2}$ as a Function of Distal Ratio (DR), Distance of Comparison (in Centimeters), and Proximal Ratio (PR)

\begin{tabular}{|c|c|c|c|c|}
\hline \multirow[b]{2}{*}{ Distance } & \multicolumn{2}{|c|}{ Group St2* } & \multicolumn{2}{|c|}{ Group St4** } \\
\hline & PR & RT & PR & RT \\
\hline \multicolumn{5}{|c|}{ Comparison Stimulus $1(\mathrm{DR}=.667)$} \\
\hline 200 & .667 & 505 & 1.333 & 559 \\
\hline 250 & .533 & 499 & 1.067 & 522 \\
\hline 300 & .444 & 501 & .889 & 474 \\
\hline 350 & .381 & 508 & .762 & 471 \\
\hline 400 & .333 & 523 & .667 & 484 \\
\hline \multicolumn{5}{|c|}{ Comparison Stimulus $2(D R=.833)$} \\
\hline 200 & .833 & 683 & 1.667 & 722 \\
\hline 250 & .667 & 597 & 1.333 & 657 \\
\hline 300 & .556 & 567 & 1.111 & 593 \\
\hline 350 & .476 & 547 & .952 & 538 \\
\hline 400 & .417 & 527 & .833 & 492 \\
\hline \multicolumn{5}{|c|}{ Comparison Stimulus $4(\mathrm{DR}=.857)$} \\
\hline 200 & .857 & 491 & .429 & 485 \\
\hline 250 & 1.071 & 496 & .536 & 519 \\
\hline 300 & 1.286 & 562 & .643 & 557 \\
\hline 350 & 1.500 & 654 & .750 & 635 \\
\hline 400 & 1.714 & 681 & .857 & 707 \\
\hline \multicolumn{5}{|c|}{ Comparison Stimulus $5(\mathrm{DR}=.750)$} \\
\hline 200 & .750 & 480 & .375 & 489 \\
\hline 250 & .938 & 482 & .469 & 484 \\
\hline 300 & 1.125 & 497 & .563 & 493 \\
\hline 350 & 1.312 & 559 & .656 & 543 \\
\hline 400 & 1.500 & 578 & .750 & 578 \\
\hline
\end{tabular}

*Overall mean $R T=547$.

**Overall mean $R T=550$.

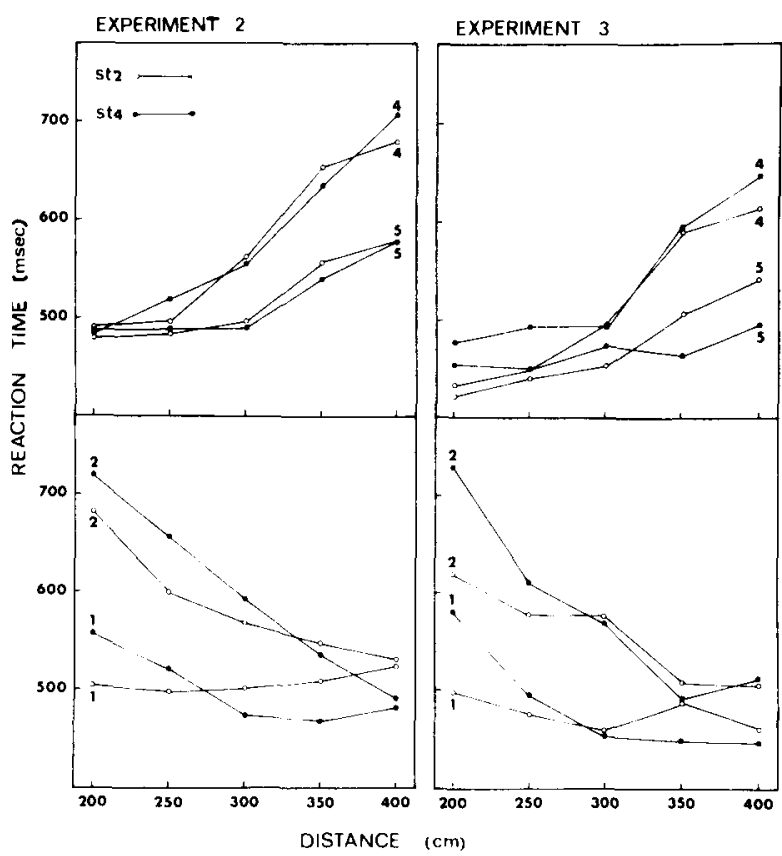

Figure 2. Mean reaction time as a function of distance of comparison stimulus for four stimulus sizes (1 and 2 in lower graph, 4 and 5 in upper graph).

of comparison square. The position of the standard did not yield a significant main effect $[F(1,22)<1]$ or a significant interaction with size of comparison $[F(3,66)<1]$. It did, however, yield a significant interaction with distance of comparison $[F(4,88)=$ $4.79, \mathrm{p}<.005]$. This appears to be due to the fact that the slopes for comparison stimuli 1 and 2 were larger in Group St4 than in Group St2. Distance of comparison yielded a significant main effect $[F(4,88)=13.66, p<.001]$ in spite of the obvious interaction with size of comparison seen in comparing the upper and lower portions of Figure 2. This interaction is, of course, significant $[F(12,264)$ $=50.46, \mathrm{p}<.001]$. The main effect of comparison size is also significant $[\mathrm{F}(3,66)=30.41, \mathrm{p}<.001]$, since sizes 2 and 4 yield higher means than sizes 1 and 5. To gain further insights into the effects of size, this variable was broken down into two binary variables: size relative to standard, larger or smaller, and similarity to standard (distal ratio), higher and lower. Thus, for example, comparison size 5 is larger than the standard and lower in similarity (lower distal ratio). Relative size does not yield a significant effect $[F(1,23)<1]$, and the means for 1 and 2 combined are virtually identical to those of 4 and 5 combined. As can be seen in the figure, this variable interacts with distance $[\mathrm{F}(4,92)=72.66, \mathrm{p}<.001]$. For sizes 1 and 2, there is an overall decrease in reaction time with distance; for sizes 4 and 5 , there is an increase. The similarity of the comparison to 
the standard yields a highly significant effect $[F(1,23)$ $=123.93, \mathrm{p}<.001]$; that is, the reaction times for stimuli 2 and 4 are much larger than those for 1 and 5 . This variable does not interact significantly with comparison distance $[F(4,92)=1.27]$ because the slopes are of opposite sign. But when this is broken down into the three-way interaction between distance and the two binary variables, a significant effect is found $[F(4,92)=44.45, p<.001]$.

To summarize, it is clear from the data that the egocentric distance plays an important role in determining the reaction times, but in dissimilar ways for the two stimuli larger than the standard and for the two smaller than the standard. The two large stimuli yield increasing reaction times with distance. The two smaller stimuli, with the exception of stimulus 1 in Group St2, yield decreasing reaction times. It does not seem to matter where the standard is placed: The functions are quite similar whether the standard is at a distance of $200 \mathrm{~cm}$ or at a distance of $400 \mathrm{~cm}$. Finally, it is seen that the slope of the reaction time function is steeper, the greater the distal ratio, that is, the greater the physical similarity between the comparison and standard stimuli.

\section{EXPERIMENT 3}

This experiment explored the effect of removing the highly textured surface on the size-judgment reaction times. There were two reasons for doing this. First, Experiment 2 had indicated that the reaction times were often related to the distance of a given comparison stimulus. It is commonly held that texture serves as an important cue to distance, and its removal might impair the subject's ability to perceive or register stimulus distance, causing an increase in, or new pattern of, reaction times. Second, occluded or intercepted texture might serve as a direct cue to size. Would the size judgment be impaired when the texture was not available? The textured cloth covering the table was replaced by a gray textureless cloth. The painted backdrop was replaced by a gray one. In all other respects, this experiment was a replication of Experiment 2, using half the number of subjects.

\section{Method}

Subjects. Twelve undergraduates at the University of Haifa, with normal or corrected vision, participated in the experiment, fulfilling a requirement of an introductory psychology course. None had participated in Experiment 1 or Experiment 2.

Apparatus. All aspects of the apparatus were identical to those of Experiment 2.

Stimulus conditions. The procedure was identical to that of Experiment 2. The two groups of subjects, St2 and St4, consisted of six subjects each.
}

\section{Results and Discussion}

The results of this experiment are virtually identical to those of Experiment 2. This can be seen in
Table 2

Mean Reaction Times (in Milliseconds) in Experiment 3 as a Function of Distal Ratios (DR) and Distance of Comparison (in Centimeters)

\begin{tabular}{|c|c|c|}
\hline \multirow[b]{2}{*}{ Distance } & \multicolumn{2}{|c|}{ Reaction Time } \\
\hline & Group St2* & Group St $4 * *$ \\
\hline \multicolumn{3}{|c|}{ Variable Stimulus $1(\mathrm{DR}=.667)$} \\
\hline 200 & 499 & 582 \\
\hline 250 & 477 & 494 \\
\hline 300 & 465 & 463 \\
\hline 350 & 489 & 451 \\
\hline 400 & 463 & 448 \\
\hline \multicolumn{3}{|c|}{ Variable Stimulus $2(\mathrm{DR}=.833)$} \\
\hline 200 & 618 & 731 \\
\hline 250 & 576 & 612 \\
\hline 300 & 578 & 567 \\
\hline 350 & 511 & 495 \\
\hline 400 & 510 & 515 \\
\hline \multicolumn{3}{|c|}{ Variable Stimulus $4(\mathrm{DR}=.857)$} \\
\hline 200 & 435 & 477 \\
\hline 250 & 447 & 494 \\
\hline 300 & 498 & 494 \\
\hline 350 & 590 & 594 \\
\hline 400 & 615 & 646 \\
\hline \multicolumn{3}{|c|}{ Variable Stimulus $5(\mathrm{DR}=.750)$} \\
\hline 200 & 423 & 454 \\
\hline 250 & 440 & 447 \\
\hline 300 & 454 & 477 \\
\hline 350 & 504 & 465 \\
\hline 400 & 540 & 496 \\
\hline
\end{tabular}

*Overall mean $R T=507 . \quad$ ** Overall mean $R T=520$.

Table 2, which presents the means of medians as a function of comparison stimulus and distance. This table is similar to Table 1, with the omission of the respective proximal ratio value, and its inspection points clearly to the correspondence between the two experiments. The correlation between mean reaction times in the equivalent stimulus conditions in the two experiments is .93. The similarity between the results of the two experiments is also evident in the two right-hand panels in Figure 2. The same analysis of variance as used in Experiment 2 was run on the data of this experiment. Its results were identical to those of Experiment 2; all effects that were significant in Experiment 2 were also significant in this experiment, and all those that were not significant there were also not significant here. Thus, the results appear to indicate that a textured background is of little importance in the size-judgment task used here.

\section{GENERAL DISCUSSION}

Three aspects of the results yield insights into the mechanisms underlying size perception. These three are, in the order in which they will be discussed below: (1) the finding that the distance of the standard square in Experiments 2 and 3 did not affect the results in any very appreciable manner; 
(2) the finding that the elimination of texture did not influence the pattern of the reaction time data; and (3) the finding of an interaction between the size of the distal ratio and the effectiveness of the distance manipulation.

Both Experiment 2 and Experiment 3 yielded quite similar results for Groups St2 and St4, in spite of the fact that the standard stimuli were placed at 200 and $400 \mathrm{~cm}$, respectively. This was surprising in view of the fact that the proximal size of the standard in Group St2 was twice that of Group St4. It appears that the subjects, knowing that the standard would not change throughout the experiment, were not making comparisons with an actual physical standard, but with some internalized representation of it. The similar performance of the two groups indicates that they utilized similar representations. One possibility is that the representation used more closely corresponded to the standard placed in the middle of the range of distances, at $300 \mathrm{~cm}$, rather than at the two extreme positions. And, indeed, when new proximal ratios were calculated, assuming that the standard had been placed at $300 \mathrm{~cm}$, they yielded a correlation of .87 with the mean reaction times of Experiment 2, considerably higher than the correlation with the originally calculated proximal ratios, .52. This, of course, explains the poor showing of the proximal ratio variable in Experiments 2 and 3 in contrast with Experiment 1. In Experiment 1 , both squares were changed on every trial and comparisons with internal representations were not possible.

The similarity of the results of Experiments 2 and 3 indicates that textured surface and background are not necessary for a size judgment task of the kind used in these experiments. Since distance was seen to play a role in most of the stimulus conditions of these two experiments, it appears that texture gradient is not a necessary cue to distance (although it is most probably a sufficient one). What distance cues were available to subjects in Experiment 3? The distances of the stimuli and the speed of the reaction times argue against the possibility that accommodation served as a cue. A more plausible alternative is a cue that has been somewhat neglected in several recent texts but received note in many older ones (e.g., Carr, 1935, p. 270; Pillsbury, 1916, p. 299)-the height, or elevation, in the visual field. The subjects viewed the scene from $15 \mathrm{~cm}$ above the table top, and every time the shutter opened, a Lilliputian microcosm revealed itself. It was as if the subjects were viewing a model of a scene in which all dimensions were reduced by about $1 / 10$ th.

The most important finding in Experiments 2 and 3 was that distance affects the reaction times to a greater extent when the comparison stimulus is more similar in size to the standard stimulus (see Figure 2).
Table 3

Reaction Times (in Milliseconds) in Experiment 1 as a

Function of Stimulus Pair and Distal Ratio

\begin{tabular}{ccccc}
\hline \multirow{2}{*}{$\begin{array}{c}\text { Stimulus } \\
\text { Pair }\end{array}$} & $\begin{array}{c}\text { Distal } \\
\text { Ratio }\end{array}$ & Mean & SD & Range \\
\cline { 3 - 5 } & .857 & 663 & 113 & 331 \\
$3-4$ & .833 & 659 & 102 & 309 \\
$2-3$ & .833 & 76 & 221 \\
$1-2$ & .800 & 623 & 56 & 180 \\
$2-4$ & .714 & 548 & 70 & 224 \\
$1-3$ & .667 & 561 & 24 & 80 \\
$1-4$ & .571 & 518 & 24 \\
\hline
\end{tabular}

In other terms, the closer the distal ratio is to 1.0 , the greater the effect of distance on the reaction times. Is this also true for the results of Experiment 1? The four square sizes in that experiment yielded six distal ratios, the six possible pairings of the four squares. Since distance should have a smaller effect for the lower distal ratios, one would expect less variability in the reaction times as the distal ratio decreases. This can be seen to be the case in Table 3, which presents the means, standard deviations, and ranges of the means for the stimulus conditions with the same distal ratio. The standard deviations and ranges of the reaction times decrease fairly systematically with decreasing distal ratios. Thus, in all three experiments the effectiveness of the distance manipulation is contingent upon the similarity of the distal sizes of the squares being compared.

This contingency between the relative size of the stimuli being compared and the effectiveness of the distance manipulation is specifically related to the direct-indirect issue. If one accepts the distinction between direct and indirect perception of size in terms of whether or not distance is taken into account, these findings appear to indicate that size perception becomes more and more indirect, the more difficult the size discrimination required. The converse of this statement is worth noting: The greater the discriminability between the sizes compared, the more direct the perception of their size. Is there evidence for direct perception of size in the three experiments? Or, in other words, are their any stimulus conditions in which distance appears not to play a role in determining the size judgment reaction times? This seems to be the case for stimulus size 1 in Group St 2 in both Experiment 2 and Experiment 3. As can be seen in Figure 2, in both experiments the reaction time/distance function for this condition is roughly parallel to the abscissa, with slopes of .09 and -.12 for Experiments 2 and 3, respectively. Neither differs significantly from zero, while all the other slopes for all other stimulus conditions in both experiments do $(\alpha=.05)$.

Separate analyses of the reaction time data of individual subjects indicate that these zero slopes are 
not the result of the averaging of diverse results. The regressions for each of the 18 subjects in Group St 2 (combining Experiments 2 and 3 ) for each of the four comparison sizes were calculated. The median for size 1 was .04 , and only three subjects yielded significant regressions (but two of these were positive, the opposite of what would be expected if distance were taken into account). In contrast, sizes 2,4 , and 5 yielded median regressions of -.66 , 1.15 , and .60 , respectively, and 42 of the 54 regressions were significant. It might be contended that the zero slopes are simply manifestations of a "floor effect." But the fact that the mean reaction time is approximately $500 \mathrm{msec}$, which is greater than binary choice reaction times in other tasks (see Teichner \& Krebs, 1974), indicates that the zero slopes are not due to response, or motor, limitations. They might, perhaps, represent some minimum on size judgment tasks. But there are several indications that this also is not the case. For example, the mean for size 5 at $200 \mathrm{~cm}$ in Group St2 in both experiments (461 msec) is significantly faster than the mean for size $1(498 \mathrm{msec})[\mathrm{t}(17)=3.60, \mathrm{p}<.01]$. Furthermore, as can be seen in Figure 2, the means for size 1 in Groups St 2 and St 4 are approximately equal, but Group St 4 yields significant negative regression slopes. Therefore, it is suggested that these zero slopes are evidence for direct size perception in one of the stimulus conditions.

If this claim for direct perception of size is correct, what information or invariant ratios in the optic array make it possible? Gibson $(1959,1979)$ proposed that the amount of texture occlusion or interception might serve this purpose, but the similarity of the results of Experiments 2 and 3 does not support this suggestion. The texture-scale size cue is probably not the primary higher order stimulus cue allowing for the direct perception of size. An alternative invariant ratio is available in the optic array, the ratio of an object's proximal size and its angle of depression from the imaginary eye-level plain. When one views a flat horizontal receding surface, there is an invariant relationship between how far below "straight ahead" a given object is in contact with that surface and the size of its retinal projection, its proximal size. Gibson (1979) makes a similar suggestion in his discussion of an experiment he carried out many years ago on size constancy for stakes planted in a plowed field. "For any distance the proportion of the stake extending above the horizon to that extending below the horizon was invariant"' (p. 160). This Gibson calls the "horizon ratio relation." In the present conceptualization, the actual horizon need not be visible, and could be occluded by surface deformations beyond the object in question, presumably without affecting the perception of its size. The results of the present experiments indicate that direct perception occurs only for relatively large differences in size, perhaps because the observer has only a rather coarse indication of the eye-level plane. It is possible that direct perception could have been attained with smaller differences in size if other ancillary information were available to the subjects, such as replacing the monocular static view with a binocular dynamic one.

The suggestion that some aspect of distance from the eye-level plane affords the direct perception of size raises some interesting implications. Distance from eye-level plane and height in the visual field are virtually identical concepts. (The former, perhaps, better demarcates an angular or linear extent that serves as a distance-invariant size metric.) Height in the visual field is also believed to be a distance cue. Thus, this cue appears to play a dual role, serving both direct and indirect perception of size. This dual role of the same cue raises some doubts about the direct-indirect dichotomy. Perhaps, rather than a dichotomy, it would be better to posit a continuum with direct size perception at one extreme and becoming more and more indirect as one moves to the other extreme. In other words, very easy size discrimination tasks require no additional processing of stimulus information, that is, direct perception. But, as the size discrimination task becomes more and more difficult, the primary direct mechanism needs more and more support from subsidiary indirect mechanisms. These yield longer and longer reaction times.

In conclusion, the results of the present set of experiments indicate that both direct and indirect size perception occur. It is further suggested that these two modes of size perception constitute a continuum from the direct to the extreme indirect mode. The easier the size discrimination, the more direct the perception, that is, the less the need for additional supporting indirect mechanisms. In the present study, the difficulty of the discrimination is expressed in terms of the distal ratio. Only the lowest distal ratio yields what appears to be direct perception of size, indifferent to stimulus distance. The indifference of the results of Experiments 2 and 3 to the position of the standard stimulus indicates that the subjects were using an internalized representation of the standard stimulus, normalized with respect to the range of distances employed. The similarity of the results of Experiments 2 and 3 indicates that direct perception of size does not rely on texture occlusion or interception. An alternative source of direct size information in the optic array is suggested: some aspect of the distance between the object's point of contact with the receding surface and the eye-level plane.

\section{REFERENCE NOTE}

1. Sternberg, S. Four theories of the effect of distance on apparent size. Paper presented at the annual meeting of the Psychonomic Society, St. Louis, October 1966. 


\section{REFERENCES}

ВЕск, J. Surface color perception. Ithaca: Cornell University Press, 1972.

Broota, K. O., \& Epstein, W. The time it takes to make veridical size and distance judgments. Perception \& Psychophysics, 1973, 14, 358-364.

CArLson, V. R. Instructions and perceptual constancy judgments. In W. Epstein (Ed.), Stability and constancy in visual percep. tion. New York: Wiley, 1977.

CARR, H. A. An introduction to space perception. New York: Longmans, Green, 1935.

EPSTE IN, W. Attitudes of judgment and the size-distance invariance hypothesis. Journal of Experimental Psychology, 1963, 66, 78-83.

EPSTE IN, W. The process of 'taking-into-account' in visual perception. Perception, 1973, 2, 267-285.

EPSTE IN, W. Observations concerning the contemporary analysis of the perceptual constancies. In W. Epstein (Ed.), Stability and constancy in visual perception. New York: Wiley, 1977.

Epste in, W., \& Broota, K. D. Attitude of judgment and reaction time in estimation of size at a distance. Perception \& Psychophysics, 1975, 18, 201-204.

Gibson, J. J. Perception as a function of stimulation. In S. Koch (Ed.), Psychology: A study of a science (Vol. 1). New York: McGraw-Hill, 1959.

Gibson, J. J. The senses considered as perceptual systems. Boston: Houghton Mifflin, 1966.

GiBson, J. J. The ecological approach to visual perception. Boston: Houghton Mifflin, 1979.
Helmholtz, H. von Treatise on physiological optics (trans. J. C. P. Southall). Rochester, N.Y: Optical Society of America, 1925.

Hochbe rg, J. Perception: II. Space and movement. In J. Kling \& L. Riggs (Eds.), Woodworth and Schlosberg's Experimental psychology. New York: Holt, Rinehart and Winston, 1971.

Hochвe RG, J. Higher-order stimuli and interresponse coupling in the perception of the visual world. In R. B. MacLeod \& H. L. Pick, Jr. (Eds.), Perception: Essays in honor of James J. Gibson. Ithaca: Cornell University Press, 1974.

MACE, W. M. James J. Gibson's strategy for perceiving: Ask not what's inside your head, but what your head's inside of. In R. Shaw \& J. Bransford (Eds.), Perceiving, acting, and knowing. Hillsdale, N.J: Erlbaum, 1977.

Oyama, T. Analysis of causal relations in the perceptual constancies. In W. Epstein (Ed.), Stability and constancy in visual perception. New York: Wiley, 1977.

PIllsbury, W. B. The fundamentals of psychology. New York: Macmillan, 1916.

Rock, I. In defense of unconscious inference. In W. Epstein (Ed.), Stability and constancy in visual perception. New York: Wiley, 1977.

Shaw, R., \& Bransford, J. Introduction: Psychological approaches to the problem of knowledge. In $R$. Shaw \& J. Bransford (Eds.), Perceiving, acting and knowing. Hillsdale, N.J: Erlbaum, 1977.

Teichner, W. H., \& KreBs, M. J. Laws of visual choice reaction time. Psychological Review, 1974, 81, 75-98.

TURveY, M. T. Contrasting orientations to the theory of visual information processing. Psychological Review, 1977, 84, 67-88.

(Received for publication December 11, 1979; revision accepted July 3, 1980.) 